Grand Valley State University

ScholarWorks@GVSU

1988

\title{
Variations on the Petrie After-Effect Procedure for Measuring the Augmenter/Reducer Personality Dimension
}

Thomas R. Herzog

Grand Valley State University, herzogt@gvsu.edu

Michael C. Vargo

Grand Valley State University

Follow this and additional works at: https://scholarworks.gvsu.edu/psy_articles

\section{ScholarWorks Citation}

Herzog, Thomas R. and Vargo, Michael C., "Variations on the Petrie After-Effect Procedure for Measuring the Augmenter/Reducer Personality Dimension" (1988). Peer Reviewed Articles. 4.

https://scholarworks.gvsu.edu/psy_articles/4

This Article is brought to you for free and open access by the Psychology Department at ScholarWorks@GVSU. It has been accepted for inclusion in Peer Reviewed Articles by an authorized administrator of ScholarWorks@GVSU.

For more information, please contact scholarworks@gvsu.edu. 


\title{
VARIATIONS ON THE PETRIE AFTEREFFECT PROCEDURE FOR MEASURING THE AUGMENTER/REDUCER PERSONALITY DIMENSION
}

\author{
THOMAS R. HERZOG AND MICHAEL C. VARGO \\ Grand Valley State College
}

\begin{abstract}
In Study I the kinesthetic aftereffect was assessed by using a wide inducing block and the Petrie (1967) measurement procedure without its initial 45-min rest period. In Study II the aftereffect was examined by employing a wide inducing block and the so-called traditional procedure (Herzog and Weintraub, 1982), following repeated inductions with a narrow inducing stimulus. In both studies, aftereffect scores generally were correlated as predicted with other indexes of the augmenter/reducer personality dimension, although the magnitudes of the relationships were somewhat lower when compared to those of typical findings. Based on research to date, suggestions are offered for increasing the magnitude of relationships between the aftereffect and questionnaire measures of augmenting/reducing.
\end{abstract}

PeTRIE (1967) proposed that augmenters are individuals who consistently increase incoming stimulation and that reducers are those who consistently decrease incoming stimulation. The kinesthetic aftereffect-a change in the felt width of a test block after one rubs an inducing block that differs in width-is supposed to provide a perceptual measure of the augmenter/reducer dimension. Augmenters should have relatively high aftereffect scores; reducers, relatively low scores.

The writers thank John Verhagen for running the subjects in Study II and Daniel J. Weintraub for suggesting Study II. Requests for reprints should be sent to Thomas Herzog, Department of Psychology, Grand Valley State College, Allendale, Michigan 49401 .

Copyright $\odot 1988$ Educational and Psychological Measurement 
Research on this topic, as reviewed by Herzog, Williams, and Weintraub (1985), indicates that the link between aftereffect scores and other indexes of the augmenter/reducer dimension is sensitive to the method of measuring the aftereffect. Subjects typically make width estimates in this task by finding a point on a long tapered wedge that feels equal in width to the test block. Chances of a positive outcome are enhanced by following the so-called Petrie procedure exactly. The procedure consists of 45 minutes of rest involving complete inactivity of the fingers, followed by four sets of four ascending (i.e., starting from near the narrow end of the wedge) judgments with interpolated induction periods of 90,90 , and 120 seconds, respectively. In addition, virtually every published study reporting a positive outcome used an inducing block wider than the test block. After failing to obtain positive results employing other procedures (Herzog and Weintraub, 1977, 1982; Weintraub, Green, and Herzog, 1973), the writers recently followed the Petrie procedure exactly and obtained consistently significant correlations between aftereffect scores and various other measures of augmenting/reducing (Herzog et al., 1985).

There are two reasons for restrained enthusiasm over this positive outcome. First, as is typically true of studies with positive outcomes, the correlations were not very large. Second, as the Petrie procedure is very time-consuming and cumbersome, it is of little practical utility. The latter observation motivated the studies reported in this paper. The writers wished to explore the methodological boundaries of the aftereffect measurement procedure within which positive outcomes might still be obtained. As a number of features of the Petrie procedure contribute to reliable measurement (e.g., long rest period, seated subject, ascending judgments only), the first concern was whether or not the 45-minute rest period could be eliminated safely. If so, the practical utility of the procedure would be greatly enhanced.

In Study I, the Petrie procedure for aftereffect measurement was followed exactly with the sole exception that the initial 45-minute rest period was eliminated. Criterion measures consisted of an updated version of an augmenter/reducer questionnaire, Form G2 (devised by Herzog et al., 1985), and the Concealed-Figures Test, which yielded consistently significant correlations with aftereffect scores in the Herzog et al. (1985) study.

According to Petrie (1967), the initial 45-minute rest period is essential because it allows all residual stimulation of the fingertips to dissipate. This dissipation presumably brings each subject to an individual basal fingertip stimulation level and allows true individual 
differences in induction to be measured from this baseline level. In Study II, the issue was whether other procedures that bring each subject to a uniquely determined starting level would also work. There is evidence that repeated inductions with the same inducing block drive individuals to uniquely determined asymptotic levels of width estimation (Herzog and Weintraub, 1977; Weintraub, Green, and Herzog, 1973). In Study II, subjects were given repeated inductions with a narrow inducing stimulus. Thus, they were driven to unique ceiling levels of width estimation. Then the aftereffect was measured by using a wide inducing stimulus.

\section{Method}

\section{Subjects}

In both studies, the subjects were undergraduate volunteers from Grand Valley State College who received extra credit in their introductory psychology course for participation. In Study I, there were 107 subjects, 36 males and 71 females. In Study II, there were 71 subjects, 40 males, 30 females, and one subject whose sex was not recorded.

\section{Apparatus}

In Study I, the induction apparatus consisted of two wooden blocks (with parallel sides) and a wooden comparison wedge. The wedge varied in width from $12.7 \mathrm{~mm}$ to $101.6 \mathrm{~mm}$. The test block was always $38.1 \mathrm{~mm}$ wide, and the inducing block was always 63.5 $\mathrm{mm}$ wide. Further specifications may be found in Herzog and Weintraub (1977). The wedge and blocks were positioned on a table in front of which the subject sat, with the wedge to the subject's left and the blocks to the subject's right. Exact positioning followed the format prescribed by Petrie (1967) as closely as possible. Subjects were blindfolded during testing. At all other times the equipment was concealed.

The revised version of the augmenter/reducer questionnaire, Form G3, was used in Study I. It consists of 50 items dealing with potential augmenter/reducer topics. The initial pool of items for Form G3 was derived from an item analysis of Form G2, using the data of Herzog et al. (1985). All items having a correlation of the same sign exceeding .15 with both induction measures from that study (composite aftereffect and residual change scores) were retained. Items not meeting this criterion but close to it were 
reworded. The remaining new items were based on the researchers' intuitions. (A few items in all versions of Form $G$ were borrowed from early versions of Zuckerman's (1978) sensation-seeking scale, a point acknowledged gratefully if somewhat belatedly.) Each item of Form G3 is a declarative statement, and subjects rate how much they agree that the item applies to them by using a 6-point scale ranging from 1 (Strongly Disagree) to 6 (Strongly Agree). Form G3 is available from the first author.

In Study II (for the data reported in this paper), the comparison wedge was the same as that in Study I. The test block was $50.8 \mathrm{~mm}$ wide and the inducing block was $63.5 \mathrm{~mm}$ wide. The test and inducing blocks were always to the left of the standing subject; the comparison wedge, always to the right. Apparatus placement and specifications were the same as those used by Herzog and Weintraub (1977). Subjects were blindfolded during testing. At all other times the equipment was concealed.

The questionnaire used in Study II (which preceded Study I chronologically) was Form G2. Its format is the same as that for Form G3. A copy of Form G2 may be found in the Appendix of a paper by Herzog et al. (1985).

\section{Procedure}

In Study I, each subject was first read some preliminary instructions in which the width-estimation task was described. The importance of permitting nothing to touch the fingertips during the measurement was stressed. The experimenter carefully monitored the subject's compliance. After the preliminary instructions, the Petrie (1967) aftereffect procedure was administered. It consisted of two ascending practice trials followed by four sets of four ascending judgments with interpolated induction periods of 90,90 , and 120 seconds, respectively. Immediately afterward, the subject was presented 14 items from the Concealed Figures Test (Psychometric Laboratory, University of North Carolina, 1951), one at a time. Each item consists of a target figure on the left and four test figures on the right that may or may not contain the target. The subject had to indicate verbally which test figures contained the target. Finally, Form G3 was given to the subject to complete.

In Study II, the aftereffect procedure, which was run first, consisted of eight induction cycles. The first four utilized vernier calipers as stimuli, with the induction stimulus set at $38.1 \mathrm{~mm}$ wide and the test stimulus at $50.8 \mathrm{~mm}$ wide. For present purposes, these induction cycles were considered preliminary inductions (data not 
reported). The results from this portion of the study may be found in Herzog (1986). The last four induction cycles utilized the blocks and wedge described in the Apparatus section. Each induction cycle consisted of four preinduction judgments of the test stimulus, a 60second induction period, and four postinduction judgments of the test stimulus. This approach, in contrast to the Petrie procedure, was named the traditional procedure by Herzog and Weintraub (1982).

For the first four induction cycles of Study II, individual judgments were made via the up-and-down method. Complete details may be found in Herzog (1986). For each judgment in the last four induction cycles, the subject held the test block with the thumb and forefinger of the left hand and simultaneously moved the thumb and forefinger of the right hand along the wedge to find the point of subjective equality. For each set of pre- and postinduction judgments, two of the judgments started from a point near the narrow end of the wedge (ascending judgments, A) and two started from a point near the wide end of the wedge (descending judgments, D). Order of ascending and descending judgments was balanced (ADDA) within each set of judgments. Further details of this procedure may be found in Herzog and Weintraub (1982).

Following the aftereffect procedure in Study II, Form G2 was given to the subject to complete. Finally, each subject was given the walking test, a measure of preferred interpersonal distance. For this test, the researcher stands against a wall and the subject stands at the entrance on the opposite side of a small room. The researcher tells the subject, "Walk up to me," and when the subject stops, the researcher places a marker at the tip of the subject's foremost foot. Later, the researcher records the distance, in inches, between the tip of the subject's foot and the tip of the researcher's foot.

\section{Scoring and Hypotheses}

For both studies, aftereffect scores were derived for each subject by subtracting the mean of the initial set of preinduction judgments from the mean of each set of postinduction judgments. The separate aftereffect scores were then averaged to procedure a composite aftereffect score for the entire procedure.

The Concealed-Figures test was scored by simply counting the number of correct choices. With 14 targets and 4 choices per target, scores could range from 0 to 56 . Scoring for interpersonal distance was described in the preceding section.

For Form G3, 20 of the 50 items were designated as those with 
which an augmenter would agree. The remaining items were considered to be those with which a reducer would agree. These designations were based on the Form G2 item analysis described earlier and the best judgment of the researchers based on their knowledge of the augmenter/reducer literature. The 30 reducer items were transformed by flipping the original rating about the midpoint of the 6point scale, which was accomplished by subtracting the original rating from 7 . Then the 50 item scores were summed to obtain an overall Augmenter Index. The scoring procedure for Form G2 was similar to that for Form G3. Details may be found in Herzog et al. (1985).

In both studies, the Form $G$ ratings were factor analyzed. Only one factor was common to both studies, a Need for Sensory Stimulation. Its scoring and relationship to aftereffect scores are to be described subsequently.

Based on the results of Herzog et al. (1985), the composite aftereffect score was expected to be positively correlated with the Form G Augmenter Index but negatively correlated with the Concealed-Figures score. The rationale for the latter prediction is that reducers should examine the geometric figures more closely than augmenters to satisfy their ongoing need for stimulation. This behavior should yield higher levels of performance for reducers and therefore a negative correlation with aftereffect scores. A positive correlation was expected between the composite aftereffect score and the interpersonal distance score. The rationale is that augmenters should be uncomfortable with the stimulation engendered by small interpersonal distances. Therefore, they would be expected to maintain relatively greater interpersonal distances than would reducers. Thus, a positive correlation with aftereffect scores would be anticipated.

\section{Results}

\section{Reliability of Measurement}

Test-retest reliability of the aftereffect was assessed by intercorrelating the separate aftereffect scores within each study after partialing out the effect of the common preinduction score. The partial correlations ranged from .68 to .74 in Study I and from .55 to .78 in Study II. These coefficients indicate reasonable test-retest reliability for the aftereffect upon immediate retesting.

It is possible to assess internal-consistency reliability for the 
aftereffect by forming two aftereffect subscale scores, each one based on half of the pre- and postinduction judgments. This approach was first proposed by Mishara and Baker (1978). Cronbach's reliability index, coefficient alpha, may be computed by using the two aftereffect subscale scores as input. Coefficient alpha was .95 for Study I and .83 for Study II. Both results replicated previous findings (Herzog et al., 1985; Mishara and Baker, 1978). Thus, internal-consistency reliability estimates were substantial for both aftereffect procedures, but higher for the Petrie procedure (minus rest period) than for the traditional measurement procedure.

Internal-consistency reliability estimates of the Concealed-Figures test and the Form $G$ Augmenter Index were assessed by computing coefficient alpha from the individual item scores for each variable. (With only one trial, it was not possible to assess the reliability of the interpersonal distance variable.) Coefficient alpha was .80 for Concealed Figures; .67 and .71 for the Augmenter Index in Studies I and II, respectively. These coefficients exceeded the minimum acceptable value of .50 suggested by Nunnally (1967).

As noted in the section on scoring, the Form $\mathrm{G}$ item ratings were factor analyzed in both studies. The purpose was to isolate any subscales of Form $G$ that appear repeatedly across studies and that might be relevant to the augmenter/reducer personality dimension. The actual procedure was a nonmetric factor analysis, the GuttmanLingoes Smallest Space Analysis III (Lingoes, 1972). Solutions in several dimensions were examined, and only factors that emerged repeatedly were retained. To be included for factor interpretation, an item had to have a factor loading of a least 1.401 on its associated factor and no loading higher than 1.351 on any other factor.

Only one factor appeared consistently in all analyses for both studies. From the four-dimensional solution, the items comprising the factor and their loadings on it are presented for both studies in Table 1. It is clear that the relevant items are either identical or very similar in both cases. Furthermore, this factor is essentially the same as one of the factors reported by Herzog et al. (1985). In all cases, the items dealt with the tendency to seek varied sensory inputs. Thus, the factor was named Need for Sensory Stimulation. A Need Sensory Stimulation score (for use in subsequent analyses) was constructed for each study by computing the mean of item scores for relevant items. Its internal-consistency reliability, assessed by computing coefficient alpha from the individual item scores for the factor, was .74 and .76 for Studies I and II, respectively. 
TABLE 1

Items and Factor Loadings for Need for Sensory Stimulation

\begin{tabular}{lc}
\hline \hline \multicolumn{1}{c}{ Item } & Loading \\
\hline Study I, Form G3: & \\
34. I enjoy parties that have lots of noise, action, and varied lighting & .71 \\
effects. & .54 \\
3. I am a "swinger." & .54 \\
40. I prefer friends who are exciting and unpredictable. & .53 \\
28. I consume more alcoholic beverages than the average person. & .52 \\
35. I need more variety and change than most people. & .43 \\
2. I crave excitement. & .59 \\
Study II, Form G2: & .58 \\
3. I am a "swinger." & .57 \\
40. I prefer friends who are exciting and unpredictable. & .55 \\
28. I drink alcoholic beverages frequently. & .52 \\
34. I enjoy parties that have lots of noise, action, and varied lighting & \\
effects. & 45 \\
29. I like to take chances. & .44 \\
2. I crave excitement. & \\
13. I enjoy the thrills of watching car races. &
\end{tabular}

\section{Personality Correlates}

Correlations between composite aftereffect scores and the criterion variables of both studies are presented in Table 2 . In the past, the authors have also presented results for a second measure of individual differences in kinesthetic induction, the residual change score (Herzog and Weintraub, 1982; Herzog et al., 1985). Because residual

TABLE 2

Product-Moment Correlations (r) Between Composite Aftereffect Scores and Criterion Variables

\begin{tabular}{lccc}
\hline \hline & \multicolumn{3}{c}{ Composite aftereffect scores } \\
\multicolumn{1}{c}{ Criterion Variables } & All & Males & Females \\
& Subjects & & \\
\hline Study I: & $.17^{*}$ & .00 & $.21^{*}$ \\
$\quad$ Form G3 augmenter (+) & $-.20^{*}$ & -.06 & $-.26^{*}$ \\
Need sensory stimulation (-) & -.08 & -.09 & -.07 \\
$\quad$ Concealed figures (-) & $.30^{* *}$ & $.26^{*}$ & $.31^{*}$ \\
Study II: & -.19 & -.14 & -.23 \\
$\quad$ Form G2 augmenter (+) & $.22^{*}$ & .25 & .23 \\
Need sensory stimulation (-) & & & \\
Interpersonal distance (+) & &
\end{tabular}

Note. The predicted relationship for each criterion variable is indicated by either $(+)$ or $(-)$. For Study I, ns = 107, 36, and 71 for all subjects, males, and females, respectively. For Study II, ns $=71,40$, and 30 for all subjects, males, and females, respectively.

${ }^{*} p<.05$, one-tailed. ${ }^{* *} p<.01$, one-tailed. 
change scores are so highly correlated with aftereffect scores (the correlation between composite aftereffect and residual change scores being .88 and .91 in Studies I and II, respectively), it was decided to dispense with results for residual change scores. In Table 2 all significance levels are one-tailed, with the predicted direction of relation indicated in parentheses for each criterion variable. Rationales for all predictions except Need for Sensory Stimulation were presented in the section on scoring. If one assumes that seeking varied sensory inputs is a strategy used by reducers to cope with their chronic boredom, then a negative correlation would be expected between Need Sensory Stimulation and aftereffect scores.

As entries in Table 2 indicate, for each study all the correlations for the entire sample were in the expected direction, and two out of three were significant. For Study II, the correlation for Need Sensory Stimulation just missed significance. If one builds a composite Need Sensory Stimulation index composed of all Form G2 items comprising this factor from either Study II or Herzog et al. (1985), the correlations between the 10-item composite index and the composite Petrie aftereffect score were $.20(p<.05)$ for all subjects; $-.08(p>.05)$ for males; and $-.37(p<.05)$ for females.

\section{Discussion}

There are two major conclusions. First, the methodological variations from the Petrie aftereffect procedure that were explored in these studies do not eliminate predicted relationships with criterion variables. Support for this conclusion comes primarily from the questionnaire variables which have now exhibited the predicted pattern of results in three studies, the two in this paper plus the one by Herzog et al. (1985). (In contrast, behavioral variables, such as Concealed-Figures performance, have proved much less consistent as criterion variables.) As replicability is commonly held to be at least as important as statistical significance in reaching conclusions, the repeated pattern of predicted relationships between the aftereffect and Form $G$ variables probably should be taken seriously. The second conclusion is that the variations from the Petrie procedure investigated in this paper do yield weakened relationships between aftereffect scores and criterion variables. This conclusion is supported by the presence of only one whole-sample validity coefficient of 1.301 or higher in the present studies compared to four in Herzog et al. (1985), where the Petrie procedure was followed exactly. Some of the decrement in correlation strength might be attributed to the 
switch from Form G2 in Herzog et al. (1985) to Form G3 in Study I. However, Form G2 was employed in Study II, which also yielded weaker correlations than those in the Herzog et al. (1985) study. A final generalization based on all three studies is that with current measures relationships between the aftereffect and criterion variables are relatively small in magnitude-sufficient for basic research but inadequate for applied diagnostic use.

Given the weak but consistent relationships between the aftereffect and questionnaire criterion variables, one may ask how such relationships could be strengthened. It is doubtful that the reliability of the Petrie aftereffect procedure (internal-consistency coefficients on the order of .90 to .95) can be increased very much. Future effort probably should be directed toward making the procedure more convenient while maintaining its reliability and validity. Perhaps preliminary inductions with a narrow inducing stimulus-an approach with demonstrated validity when used with the traditional aftereffect procedure (Study II)—can be substituted for the initial rest period in the Petrie procedure to produce an optimal combination of reliability, validity, and convenience.

The most promising hope for strengthening aftereffect-criterion relationships probably lies with an improved questionnaire. Item analyses and factor analyses of Form $G$ from the three studies yielding positive outcomes suggest the following improvements. More items tapping a need for sensory stimulation should be included-a suggestion that dovetails nicely with the research on sensation seeking by Zuckerman (1978). In addition, more items dealing with participation in sports, extraversion, participation in antisocial behavior, and tolerance for physical discomfort (especially pain) should be included. Items about these topics are supported not only by analyses of Form $\mathrm{G}$ but also by positive findings in the augmenter/reducer literature (e.g., Eysenck, 1962; Petrie, 1967; Ryan and Foster, 1967). Thus, a questionnaire concentrating on all of these types of items might very well yield significant increments in the aftereffect-criterion relationship.

\section{REFERENCES}

Eysenck, H. J. (1962). Figural aftereffects, personality, and intersensory comparisons. Perceptual and Motor Skills, 15, 405-406.

Herzog, T. R. (1986). The disappearing aftereffect: Fact or artifact? American Journal of Psychology, 99, 289-292.

Herzog, T. R. and Weintraub, D. J. (1977). Preserving the kinesthetic aftereffect: Alternating inducing blocks day by day. American Journal of Psychology, 90, 461-474. 
Herzog, T. R. and Weintraub, D. J. (1982). Roundup time at personality ranch: Branding the elusive augmenters and reducers. Journal of Personality and Social Psychology, 42, 729-737.

Herzog, T. R., Williams, D. M., and Weintraub, D. J. (1985). Meanwhile, back at personality ranch: The augmenters and reducers ride again. Journal of Personality and Social Psychology, 48, 1342-1352.

Lingoes, J. L. (1972). A general survey of the Guttman-Lingoes nonmetric program series. In R. N. Shepard, A. K. Romney, and S. B. Nerlove (Eds.), Multidimensional scaling, (Vol. 1). New York: Seminar Press, pp. 52-68.

Mishara, B. L. and Baker, A. H. (1978). Kinesthetic aftereffect scores are reliable. Applied Psychological Measurement, 2, 239247.

Nunnally, J. (1967). Psychometric theory. New York: McGraw-Hill.

Petrie, A. (1967). Individuality in pain and suffering. Chicago: University of Chicago Press.

Ryan, E. D. and Foster, R. (1967). Athletic participation and perceptual augmentation and reduction. Journal of Personality and Social Psychology, 6, 472-476.

Weintraub, D. J., Green, G. S., and Herzog, T. R. (1973). Kinesthetic aftereffects day by day: Trends, task features, reliable individual differences. American Journal of Psychology, 86, 827844.

Zuckerman, M. (1978, February). The search for high sensation. Psychology Today, pp. 39-46, 96-99.

Psychometric Laboratory, University of North Carolina (1951) Concealed Figures Test. Chapel Hill, NC: Author. 